\title{
Cell Polarity in Bacillus subtilis: Statistical Analysis of Factors Influencing the Positions of Spores in Sister Sporangia
}

\author{
By G. DUNN* \\ Microbiology Unit, Department of Biochemistry, University of Oxford, \\ Oxford $O X 13 Q U$
}

(Received 26 March 1979)

\begin{abstract}
The positions of spores within chains of four sporangia ('quads') of Bacillus subtilis have been determined. These data have been used to test statistical models constructed to predict the patterns of sporulation in cultures containing these quads. If the probability that a spore is formed distally in relation to the newest division septum is used as a measure of cell polarity, then a cell's polarity is influenced by the behaviour of its sister sporangium (i.e. sister cells do not position their spores independently), but not by its position within a quad. During the course of this work it was also found that cell polarity in B. subtilis is markedly affected by the ionic composition of the growth medium.
\end{abstract}

\section{INTRODUCTION}

Hitchins \& Slepecky (1969) have suggested that bacterial sporulation could be considered to be a modified cell division. The acceptance of such a view implies that one should consider a developing sporangium as a pair of differentiating sister cells, rather than as a single differentiating bacterium. At the beginning of sporulation the parent cell forms a septum near to one of its ends, rather than at the centre as in a vegetative division. The smaller of the two daughter cells (the prespore compartment) then develops into a dormant spore that is capable of germination and subsequent outgrowth. The other daughter cell (the sporemother compartment) engulfs the developing prespore, provides it with macromolecular components, and finally lyses to release the mature spore into the medium.

The cells of Gram-positive bacilli such as Bacillus subtilis usually remain attached for some time after the formation of a division septum, and during vegetative growth a culture may contain chains containing two, four, eight or more cells. If sporulation is induced in such a culture by resuspending the bacteria in a poor medium, chains of developing sporangia are produced. By looking at the distribution of spores within these chains one can then observe an example of pattern formation that is caused by two or more simultaneous asymmetric cell divisions. This is a relatively simple developmental system that can be used as a model, or analogue, of the development of pattern in a higher organism.

It is easy to determine by phase-contrast microscopy whether a spore has been formed at the 'old' (distal to the newest division septum) or 'new' (proximal to the newest division septum) end of a sporangium (Hitchins, 1975). The possible patterns of spore positions in sister sporangia are as follows (assuming that all sporangia contain phase-bright spores):
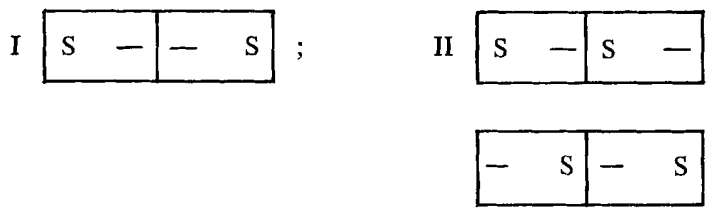

* Present address: General Practice Research Unit, Institute of Psychiatry, De Crespigny Park, Denmark Hill, Camberwell, London SE5. 
where $S$ indicates a spore, and - indicates no spore. We have previously shown that, in cultures of B. subtilis, spores in pairs of sporangia are positioned randomly, and that the probability of a spore being formed at the old end of a sporangium depends on the constitution of the growth medium (Dunn \& Mandelstam, 1977). After growth on a medium containing casein hydrolysate and salts (Sterlini \& Mandelstam, 1969), this probability is about 0.75 ; after growth on brain heart infusion broth, it is about 0.50 . If the probability value is regarded as a measure of cell polarity during growth, then polarity is clearly affected by the cells' environment.

The initiation of sporulation in sister cells of B. subtilis has been studied by Dawes et al. (1971). Here the interdependence of sister sporangia is very marked. Usually both sporangia contain spores, or neither does. It was suggested that sporulation is initiated in a growing cell at the time of resuspension, and that this cell subsequently divides to produce two developing sporangia. The implication is that the early events of sporulation occur before the final vegetative cell division. If some of these events are concerned with the positioning of the prespore septum, then one might expect some interdependence of sister sporangia with regard to the position of their spores.

The present paper gives a more detailed description of cell polarity in $B$. subtilis. This enables one to investigate any possible interdependence of sister sporangia through the construction of theoretical models that can be used to predict patterns of sporulation in chains of B. subtilis sporangia. The first requirement was to distinguish one sister sporangium from the other (so that class II above becomes two separate classes). This was accomplished by examining chains of four sporangia ('quads'). If one considers a quad, one can distinguish the two sporangia on the basis of their position:

\begin{tabular}{|l|l|l|l|}
\hline$\alpha$ & $\beta$ & $\beta$ & $\alpha$ \\
\hline
\end{tabular}

where the outer sporangia are here called type $\alpha$, and the inner, type $\beta$. A quad consists therefore of two pairs of distinguishable sporangia; each pair containing one type $\alpha$ sporangium and one type $\beta$.

When one has recorded the positions of spores within the sporangia of a quad, one can ask the following questions. (1) Is the polarity of a cell that gives rise to a type $\alpha$ sporangium different from that in a cell that gives rise to one of type $\beta$ ? That is, is there any effect of sporangial position within a quad on polarity? (2) Are sister sporangia interdependent or do they position their spores independently? For example, is there any influence of a type $\alpha$ sporangium on its sister type $\beta$ sporangium, or vice versa?

The results reported in this paper indicate that there is no difference in the polarity of the sister cells that give rise to type $\alpha$ or type $\beta$ sporangia. They do show, however, that the sister cells are to some extent interdependent. During the course of this work it was also found that the ionic content of the growth medium had a marked effect on cell polarity in cultures of $B$. subtilis.

\section{THEORY}

\section{Notation}

Consider chains of four sporangia (quads) in a sporulating culture of $B$. subtilis. Each sporangium will contain a spore either at the old end of the cell or at the new end. The analysis of the experimental data is made much easier by using the following shorthand notation to describe all possible classes of quad. A sporangium is scored as 1 if the spore is at its old end, or 0 if at the new end. The spore positions within a quad are therefore recorded as a sequence of four symbols corresponding to its four sporangia taken in order. The ten distinguishable classes of quad that contain four spores are shown in Table 1. Other classes of quad that contain fewer than four spores are not considered in this paper. 
Table 1. Shorthand notation for the classes of quad observed by phase-contrast microscopy

Class*

Notation $\dagger$

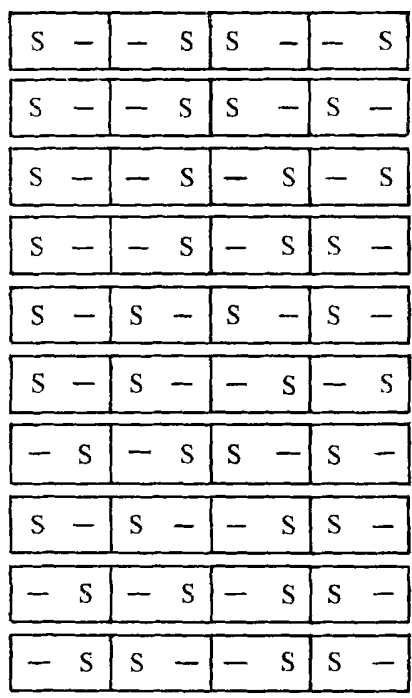

1111

1110

1101

1100

1010

1001

0110

1000

0100

0000

* $S$ indicates the presence of a spore, and - no spore.

$\dagger 1$ indicates that the spore is at an old end, and 0 that it is at the new end.

When the intracellular positions of spores are scored without regard to the position of the sporangia within the quads, the formation of a spore at an old end of a sporangium is called event $\mathrm{C}$, and the probability of this event occurring is $P(\mathrm{C})$. If the sporangia of types $\alpha$ and $\beta$ are distinguished, the formation of a spore at the old end of a type $\alpha$ sporangium is called event $\mathrm{A}$, and the associated probability is $P(\mathrm{~A})$. Similarly, the formation of a spore at the old end of a type $\beta$ sporangium is event $B$, and its probability is $P(B)$. If the spore is formed at the new end of a sporangium, this event is called $\mathrm{C}^{\prime}, \mathrm{A}^{\prime}$ or $\mathrm{B}^{\prime}$, depending on the type of sporangium being considered. By definition, $P(\mathrm{C})+P\left(\mathrm{C}^{\prime}\right)=1, P(\mathrm{~A})+P\left(\mathrm{~A}^{\prime}\right)=1$, and $P(\mathrm{~B})+P\left(\mathrm{~B}^{\prime}\right)=1$.

Now, if we consider a pair of sister sporangia within a quad, the following conditional probabilities can be defined. Given that there is a spore at the old end of the type $\alpha$ sporangium (event $A$ ), the probability of a spore being found at the old end of the sister type $\beta$ sporangium is $P(\mathrm{~B} / \mathrm{A})$. Similarly, given that there is a spore at the new end of the type $\alpha$ sporangium (event $\mathrm{A}^{\prime}$ ), the probability of finding a spore at the old end of the sister type $\beta$ sporangium is $P\left(\mathrm{~B} / \mathrm{A}^{\prime}\right)$.

For any particular culture, maximum likelihood estimates of the above probabilities can be determined from the observed frequencies of the 10 possible classes of quad. To simplify the algebraic formulae in Table 2 , the following variables are substituted for the respective values for these probabilities: $\quad p=P(\mathrm{C}), \quad q=P(\mathrm{~A}), \quad r=P(\mathrm{~B}), \quad s=P(\mathrm{~B} / \mathrm{A})$ and $t=P\left(\mathrm{~B} / \mathrm{A}^{\prime}\right)$.

\section{Statistical models}

The models were designed to answer the two questions posed in the Introduction. Does the position of a starving cell within a quad influence its polarity, and do sister cells in a quad behave independently? The models and their consequences are as follows.

(a) Behaviour of a sporangium is not influenced by its position within a quad and is independent of the behaviour of its sister sporangium. Here all the cells of a particular culture are assumed to have the same polarity. That is, the probabilities of occurrence of the various 


\section{Table 2. Probabilities of the 10 distinguishable classes of quad} that contain four spores predicted by the four theoretical models

In a chain of four sporangia there are two types of sporangia that can be distinguished by their position. Sporangia of type $\alpha$ are those at the two ends of the quad; sporangia of type $\beta$ are the two inner sporangia. In model $(a)$, the probability $p$ is the probability of observing a spore at an old end of a sporangium, irrespective of its position in the quad [i.e. $P(\mathrm{C})$ ]. In model $(b), q$ is the probability of observing a spore at the old end of a type $\alpha$ sporangium [i.e. $P(\mathrm{~A})$ ], while $r$ is the probability of observing a spore at the old end of a type $\beta$ sporangium [i.e. $P(B)$ ]. In models $(c)$ and $(d), q$ is the same as in model $(b)$. The conditional probability $s$ is that of observing a spore at an old end of a type $\beta$ sporangium given that there is a spore at the old end of its sister type $\alpha$ sporangium (i.e. $P(B / A)$ ). Similarly, $t$ is the conditional probability of observing a spore at the old end of a type $\beta$ sporangium given that there is a spore at the new end of its sister type $\alpha$ sporangium [i.e. $P\left(\mathrm{~B} / \mathrm{A}^{\prime}\right)$ ].

Predicted probabilities

\begin{tabular}{llll} 
Class & Model $(a)$ & \multicolumn{1}{c}{ Model $(b)$} & \multicolumn{1}{c}{ Models $(c)$ and $(d)$} \\
1111 & $p^{4}$ & $q^{2} r^{2}$ & $q^{2} s^{2}$ \\
1110 & $2 p^{3}(1-p)$ & $2 q r^{2}(1-q)$ & $2 q s(1-q) t$ \\
1101 & $2 p^{3}(1-p)$ & $2 q^{2} r(1-r)$ & $2 q^{2} s(1-s)$ \\
1100 & $2 p^{2}(1-p)^{2}$ & $2 q r(1-q)(1-r)$ & $2 q s(1-q)(1-t)$ \\
1010 & $2 p^{2}(1-p)^{2}$ & $2 q r(1-q)(1-r)$ & $2 q(1-s)(1-q) t$ \\
1001 & $p^{2}(1-p)^{2}$ & $q^{2}(1-r)^{2}$ & $q^{2}(1-s)^{2}$ \\
0110 & $p^{2}(1-p)^{2}$ & $r^{2}(1-q)^{2}$ & $t^{2}(1-q)^{2}$ \\
1000 & $2 p(1-p)^{3}$ & $2 q(1-q)(1-r)^{2}$ & $2 q(1-q)(1-t)(1-s)$ \\
0100 & $2 p(1-p)^{3}$ & $2 r(1-q)^{2}(1-r)$ & $2 t(1-q)^{2}(1-t)$ \\
0000 & $(1-p)^{4}$ & $(1-q)^{2}(1-r)^{2}$ & $(1-q)^{2}(1-t)^{2}$
\end{tabular}

classes of quad can be predicted from the experimentally determined estimate of a single parameter, $P(\mathrm{C})$. These probabilities are listed in Table 2 . This model implies the equality of the following probabilities: $P(\mathrm{C}), P(\mathrm{~A}), P(\mathrm{~B}), P(\mathrm{~B} / \mathrm{A})$ and $P\left(\mathrm{~B} / \mathrm{A}^{\prime}\right)$.

(b) Behaviour of a sporangium is influenced by its position within a quad, but is independent of the behaviour of its sister sporangium. Here the probability that a spore will be found at the old end of a type $\alpha$ sporangium, $P(\mathrm{~A})$, is assumed to be different to that of finding a spore at the old end of a type $\beta$ sporangium, $P(B)$. In this case, the probabilities of occurrence of the different classes of quad can be predicted from the experimentally determined estimates of $P(\mathrm{~A})$ and $P(\mathrm{~B})$. These probabilities are listed in Table 2 . Independence of a sister sporangium implies that $P(\mathrm{~B} / \mathrm{A})=P\left(\mathrm{~B} / \mathrm{A}^{\prime}\right)$.

(c) Behaviour of a sporangium is independent neither of its position within a quad nor of the behaviour of its sister sporangium. This model implies that $P(\mathrm{~A})$ should not equal $P(\mathrm{~B})$, and that $P(\mathrm{~B} / \mathrm{A})$ should not equal $P\left(\mathrm{~B} / \mathrm{A}^{\prime}\right)$. The probabilities of occurrence of the different classes of quad can be predicted from the experimentally determined estimates of $P(A)$, $P(\mathrm{~B} / \mathrm{A})$ and $P\left(\mathrm{~B} / \mathrm{A}^{\prime}\right)$, and they are given in Table 2 .

(d) Behaviour of a sporangium is independent of its position within a quad, but not of the behaviour of its sister sporangium. As in model $(c)$ the probabilities of occurrence of the different classes of quad can be predicted from the values of $P(\mathrm{~A}), P(\mathrm{~B} / \mathrm{A})$ and $P\left(\mathrm{~B} / \mathrm{A}^{\prime}\right)$. These frequencies of quad cannot, therefore, be used to distinguish models $(c)$ and $(d)$. However, model $(d)$ differs from model $(c)$ in implying the equality of $P(\mathrm{~A})$ and $P(\mathrm{~B})$.

\section{METHODS}

Strains and culture conditions. Bacillus subtilis 168 (trpC2) was used in the initial experiments (Dunn \& Mandelstam, 1977) and is referred to as the wild-type. Bacillus subtilis strain FJ3 (lyt metC3) is a mutant deficient in an autolytic enzyme and also has a requirement for methionine; it grows as long chains of unseparated cells (Fein \& Rogers, 1976). These strains were grown in one of four media: (1) a hydrolysed casein and salts medium (salts-CH), previously used by Sterlini \& Mandelstam (1969); (2) a medium containing $1 \%(\mathrm{w} / \mathrm{v})$ casein hydrolysate (Oxoid), $0.45 \%(\mathrm{w} / \mathrm{v})$ sodium glutamate and $0.05 \mathrm{M}-\mathrm{MgCl}_{\mathbf{2}}(\mathrm{Mg}-\mathrm{CH})$; (3) a medium similar to (2) except that it contained $0.05 \mathrm{M}-\mathrm{K}_{2} \mathrm{SO}_{4}$ instead of $\mathrm{MgCl}_{2}(\mathrm{~K}-\mathrm{CH})$; and (4) brain 
Table 3. Estimates of the parameters of the statistical models

Each set of estimates was obtained from the results of a single experiment. The experimental results for the wild-type are given in Tables 4 and 5. The wild-type was grown on salts-CH or on BHIB. Strain FJ3 was grown on salts-CH, BHIB, Mg-CH or $\mathrm{K}-\mathrm{CH}$. In all experiments the bacteria were resuspended in a poor medium containing glutamate and salts to induce sporulation. See Table 2 for definitions of probabilities.

$\begin{array}{lcccccc}\text { Probability } & \begin{array}{c}\text { Estimate } \\ \text { (salts-CH) }\end{array} & \begin{array}{c}\text { Wild-type } \\ \text { (BHIB) }\end{array} & \begin{array}{c}\text { FJ3 } \\ \text { (salts-CH) }\end{array} & \begin{array}{c}\text { FJ3 } \\ (\mathrm{BHIB})\end{array} & \begin{array}{c}\text { FJ3 } \\ (\mathrm{Mg}-\mathrm{CH})\end{array} & \begin{array}{c}\text { FJ3 } \\ (\mathrm{K}-\mathrm{CH})\end{array} \\ P(\mathrm{C}) & 0.797 & 0.557 & 0.90 & 0.84 & 0.52 & 0.80 \\ P(\mathrm{~A}) & 0.824 & 0.480 & 0.91 & 0.86 & 0.51 & 0.82 \\ P(\mathrm{~B}) & 0.770 & 0.633 & 0.89 & 0.83 & 0.53 & 0.77 \\ P(\mathrm{~B} / \mathrm{A}) & 0.780 & 0.649 & 0.90 & 0.86 & 0.55 & 0.77 \\ P\left(\mathrm{~B} / \mathrm{A}^{\prime}\right) & 0.720 & 0.612 & 0.77 & 0.61 & 0.50 & 0.73\end{array}$

heart infusion broth with $0.2 \%$ (w/v) yeast extract (Difco) (BHIB). Cultures were shaken at $37^{\circ} \mathrm{C}$ and harvested while in exponential growth $\left(0.25 \mathrm{mg}_{\text {dry }} \mathrm{wt} \mathrm{ml}^{-1}\right)$; sporulation was induced by transferring the cells to a resuspension medium containing glutamate and inorganic ions, also at $37^{\circ} \mathrm{C}$ (Sterlini \& Mandelstam, 1969). All media were supplemented with L-tryptophan $\left(20 \mu \mathrm{g} \mathrm{ml}^{-1}\right)$ for the wild-type or L-methionine (200 $\mu \mathrm{g} \mathrm{ml}^{-1}$ ) for mutant FJ3.

Microscopy. Samples were removed from sporulating cultures 6 to $8 \mathrm{~h}$ after resuspension and stored in a $10 \%$ solution of formaldehyde. The intracellular positions of phase-bright spores in chains containing four sporangia (quads) were recorded; individual sporangia and those in chains of different length were disregarded.

\section{RESULTS AND DISCUSSION}

\section{Cell polarity after growth on salts-CH or $B H I B$}

In the first two experiments, the wild-type was grown on salts- $\mathrm{CH}$ or on BHIB and then resuspended in the glutamate-salts medium to induce sporulation. In both cultures quads were quite common (about $20 \%$ of the population). This is not always the case with cultures of the wild-type, and this is a disadvantage of using this strain. About 2500 quads were scored in the culture that had grown in salts- $\mathrm{CH}$, and about 900 in the BHIB culture. The frequencies of the 10 distinguishable classes of quad are given in Tables 4 and 5 respectively. The estimates of probabilities that were determined from these observed frequencies are given in Table 3. As reported by Dunn \& Mandelstam (1977), the patterns of sporulation in these two cultures are clearly different.

In two further experiments, strain FJ3 was induced to sporulate after growth in salts- $\mathrm{CH}$ or in BHIB and about 200 quads were scored. The results are summarized in Table 3. In $\mathrm{FJ} 3$, in contrast to the wild-type, cell polarity in BHIB appeared to be little different from that in salts-CH. However, the value of $P\left(\mathbf{B} / \mathbf{A}^{\prime}\right)$ was lowered by growth in BHIB. Each of these two experiments was repeated a further four times and the mean values for the estimates of $P(\mathrm{~B} / \mathrm{A})$ and $P\left(\mathrm{~B} / \mathrm{A}^{\prime}\right)$ were determined. After growth on salts $-\mathrm{CH}$, the mean value of $P(\mathrm{~B} / \mathrm{A})$ was 0.80 (standard deviation 0.11 ) and that of $P\left(\mathrm{~B} / \mathrm{A}^{\prime}\right)$ was 0.71 (standard deviation 0.13 ). After growth in BHIB, the corresponding values were 0.82 (standard deviation 0.03 ) and 0.61 (standard deviation 0.07 ), respectively.

\section{Cell polarity after growth on $\mathrm{Mg}-\mathrm{CH}$ or $\mathrm{K}-\mathrm{CH}$}

Preliminary experiments in which the wild-type was grown in mixtures of salts- $\mathrm{CH}$ and BHIB indicated that the salts- $\mathrm{CH}$ medium might contain something that was altering cell polarity. Further experiments were carried out to check whether cell polarity could be influenced by the ionic composition of the growth medium. A medium was devised that contained $1 \%(\mathrm{w} / \mathrm{v})$ casein hydrolysate (the same as that contained in the medium of Sterlini \& Mandelstam) and $0.45 \%(\mathrm{w} / \mathrm{v})$ sodium glutamate, with $20 \mu \mathrm{g} \mathrm{L}$-tryptophan $\mathrm{ml}^{-1}$ or 


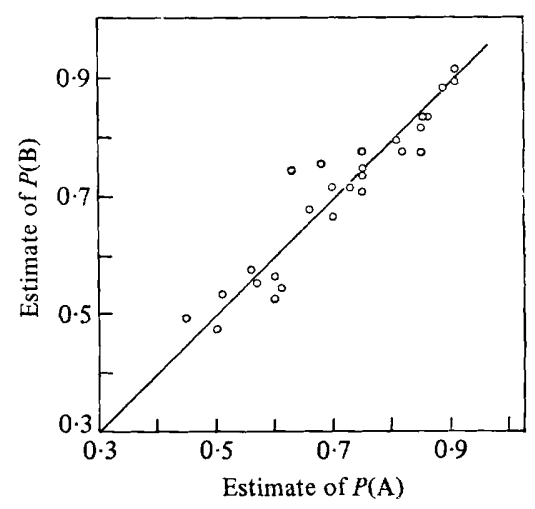

Fig. 1. Relationship between estimates of $P(\mathrm{~A})$ and $P(\mathrm{~B})$ in strain $\mathrm{FJ3}$. Changes in polarity were induced by changing the composition of the growth medium (see text). The scatter diagram contains data from 29 experiments. The line drawn is that expected if the two parameters were identical. The estimated value of the correlation coefficient $(\rho)$ is 0.95 . See text for the definition of $P(\mathrm{~A})$ and $P(\mathrm{~B})$.

$200 \mu \mathrm{g}$ L-methionine $\mathrm{ml}^{-1}$ added when necessary. Both the wild-type and strain FJ3 grew on this medium with a mean generation time (about $40 \mathrm{~min}$ ), that is, the same as that obtained with the salts-CH medium of Sterlini \& Mandelstam (1969).

The medium was then supplemented with inorganic salts in an attempt to influence cell polarity. Two of the modified media that were particularly useful for the analysis of cell polarity were $\mathrm{Mg}-\mathrm{CH}$ and $\mathrm{K}-\mathrm{CH}$. The former contained $0.05 \mathrm{M}-\mathrm{MgCl}_{2}$ and the latter $0.05 \mathrm{M}-$ $\mathrm{K}_{2} \mathrm{SO}_{4}$. In five experiments in which the wild-type was grown in $\mathrm{K}-\mathrm{CH}$ and then induced to sporulate, about 200 pairs of sporangia in each experiment were scored for their spore positions. The mean value for the probability of observing a spore at an old cell end was 0.80 (standard deviation 0.04 ). In 13 similar experiments in which the wild-type was grown on $\mathrm{Mg}-\mathrm{CH}$, the mean value for this probability was 0.48 (standard deviation $0 \cdot 10$ ). The results indicate that the change in polarity which is typical of a shift from salts- $\mathrm{CH}$ to BHIB can be mimicked by replacing $\mathrm{K}_{2} \mathrm{SO}_{4}$ by $\mathrm{MgCl}_{2}$ in the same hydrolysed casein medium.

An unfortunate characteristic of the sporulating cultures of the wild-type that had grown on $\mathrm{Mg}-\mathrm{CH}$ was that they contained a very small proportion of quads or of longer chains. To overcome this difficulty the autolytic enzyme-deficient mutant strain FJ3, which forms long chains of cells, was used in all subsequent experiments. This strain was grown either on $\mathrm{K}-\mathrm{CH}$ or $\mathrm{Mg}-\mathrm{CH}$ and then resuspended to induce sporulation. Quads were then scored for their spore positions, and the probabilities that were determined from the observed frequencies of classes of quad are given in Table 3 . In contrast to the behaviour of this strain after growth on BHIB, cell polarity was significantly reduced by growing the bacteria on $\mathrm{Mg}-\mathrm{CH}$.

\section{Relationship between parameters describing cell polarity in strain FJ3}

The purpose of the next series of experiments was to investigate the relationships between estimated values of $P(\mathrm{~A})$ and $P(\mathrm{~B})$. In all, data from 29 experiments were analysed. In each experiment strain FJ3 was grown in one of the four growth media (see Methods) and then resuspended to induce sporulation. The positions of spores in quads was then scored, and the values of $P(\mathrm{~A})$ and $P(\mathrm{~B})$ were estimated. A scatter diagram showing the relationship between the estimates of $P(\mathrm{~A})$ and $P(\mathrm{~B})$ is shown in Fig. 1.

Consider the possible differences between type $\alpha$ and type $\beta$ sporangia. Figure 1 shows a high positive correlation between the estimates of $P(\mathrm{~B})$ and $P(\mathrm{~A})$ in strain FJ3 $(\rho=0.95)$ and indicates that the relationship between the corresponding values for the two probabilities 
Table 4. Sporulation patterns in quads of the wild-type that contain four spores; after growth on salts- $\mathrm{CH}$

Models $(a)$ to $(d)$ are those given in Table 2. The values of $P(\mathrm{~A}), P(\mathrm{~B})$ etc. substituted into the expressions given in Table 2 are the experimentally determined probabilities given in Table 3. Quad types 0100 and 0000 are treated as a single class for the purpose of significance tests. The following values were determined for $x^{2}$ : model $(a), 61 \cdot 21$ (7 degrees of freedom); model $(b)$, $18 \cdot 16$ (6 degrees of freedom); models $(c)$ and $(d), 6.48$ (5 degrees of freedom).

\begin{tabular}{lcccc} 
Cbserved & \multicolumn{3}{c}{ Expected frequency } \\
Class & $\begin{array}{c}\text { Model }(a) \\
\text { frequency }\end{array}$ & Model $(b)$ & $\begin{array}{c}\text { Models } \\
(c) \text { and }(d)\end{array}$ \\
1111 & 1051 & 1001 & 999 & 1025 \\
1110 & 379 & 510 & 427 & 404 \\
1101 & 560 & 510 & 597 & 578 \\
1100 & 149 & 130 & 127 & 157 \\
1010 & 128 & 130 & 127 & 114 \\
1001 & 81 & 65 & 89 & 82 \\
0110 & 46 & 65 & 46 & 40 \\
1000 & 49 & 33 & 38 & 44 \\
0100 & 31 & 33 & 27 & 31 \\
0000 & 8 & 4 & 4 & 6
\end{tabular}

Table 5. Sporulation patterns in quads of the wild-type that contain four spores; after growth on BHIB

Models $(a)$ to $(d)$ are those given in Table 2. The values of $P(A), P(B)$ etc. substituted into the expressions given in Table 2 are the experimentally determined probabilities given in Table 3. The following values were determined for $\chi^{2}$ : model $(a), 93.32$ (8 degrees of freedom); model $(b), 7.07$ (7 degrees of freedom); models $(c)$ and $(d), 7 \cdot 35$ (6 degrees of freedom).

\begin{tabular}{lcccc} 
Class & \multicolumn{3}{c}{ Expected frequency } \\
1111 & $\begin{array}{c}\text { Observed } \\
\text { frequency }\end{array}$ & Model $(a)$ & Model $(b)$ & $\begin{array}{c}\text { Models } \\
(c) \text { and }(d)\end{array}$ \\
1110 & 86 & 84 & 80 & 84 \\
1101 & 164 & 133 & 174 & 172 \\
1100 & 107 & 133 & 93 & 91 \\
1010 & 99 & 106 & 101 & 109 \\
1001 & 88 & 106 & 101 & 93 \\
0110 & 24 & 53 & 27 & 25 \\
1000 & 96 & 53 & 94 & 88 \\
0100 & 50 & 84 & 58 & 59 \\
0000 & 115 & 84 & 109 & 112 \\
& 40 & 33 & 32 & 35
\end{tabular}

is $P(\mathrm{~B})=P(\mathrm{~A})$ There is no reason to believe that on average the position of a sporangium within a quad is important in determining its polarity. It is assumed that this conclusion also applies to wild-type sporangia.

\section{Analysis of statistical models}

Consider the wild-type after growth on salts- $\mathrm{CH}$. Table 4 gives the expected frequencies of each class of quad based on each of the statistical models. From the values of $\chi^{2}$ given in this table it is clear that models $(a)$ and $(b)$ can both be rejected (significance level $5 \%$ ), but models $(c)$ and $(d)$ cannot. This conclusion implies that sister sporangia do not position their spores independently. Table 5 shows the predicted frequencies for each class of quad after growth of the wild-type on BHIB. In this case the frequencies predicted by models 
$(b),(c)$ and $(d)$ fit the observed data equally well. Here there does not appear to be any significant interaction between sister sporangia. To summarize, the distribution of spores can be described in terms of three parameters: $P(\mathrm{~A}), P(\mathrm{~B} / \mathrm{A})$ and $P\left(\mathrm{~B} / \mathrm{A}^{\prime}\right)$. The first is an overall measure of cell polarity, the difference between the latter two a measure of cell interaction. Changes in culture conditions can affect either cell polarity, or cell interaction, or both. Data obtained from cultures of strain FJ3 (Table 3) indicate that the genotype of the bacteria can also affect both cell polarity and cell interaction.

Consider further the observed proportion of sporangia containing distal spores as measure of cell polarity in $B$. subtilis. This proportion $(P)$ can be thought of as measuring the sum of the following components: $P=\pi+\delta+\epsilon$, where $P$ is the estimated value of $P(\mathrm{~A})$ $P(\mathrm{~B})$ or $P(\mathrm{C}), \pi$ is the true underlying value of the corresponding cell polarity, $\delta$ is a measure of the unexplained variation between cultures (and, say, between the type $\alpha$ and type $\beta$ sporangia within a single culture), and $\epsilon$ is the experimental (counting) error. Both $\delta$ and $\epsilon$ are random variables with mean values equal to zero, so that an average of the values of $P$, determined from several cultures, will be an estimator of $\pi$. However, for a single culture, $P$ will be an estimator of $\pi+\delta$. For example, results from a single experiment can show that $P(\mathrm{~A})$ does not equal $P(\mathrm{~B})$, yet Fig. 1 indicates that the underlying cell polarities for the two types of sporangia are the same.

To conclude, this paper suggests a way in which the distribution of spores within chains of sporangia (and presumably the underlying cell polarity) can be described. It does not offer any physical or biochemical explanation for the patterns of sporulation. We have previously suggested that an important factor is likely to be the structure of the cell wall (Dunn \& Mandelstam, 1977), but there is no firm evidence available to support this suggestion.

This work was supported by the Science Research Council. I wish to thank Professor J. Mandelstam, Dr R. W. Hiorns and Dr B. N. Dancer for advice and criticism.

\section{REFERENCES}

Dawes, I. W., Kay, D. \& Mandelstam, J. (1971). Determining effect of growth medium on the shape and position of daughter chromosomes and on sporulation in Bacillus subtilis. Nature, London 230, 567-569.

DunN, G. \& Mandelstam, J. (1977). Cell polarity in Bacillus subtilis: effect of growth conditions on spore position in sister cells. Journal of General Microbiology 103, 201-205.

FeIN, J. E. \& Rogers, H. J. (1976). Autolytic enzymedeficient mutants of Bacillus subtilis. Journal of Bacteriology 127, 1427-1442.
Hitchins, A. D. (1975). Polarized relationship of bacterial spore loci to the 'old' and 'new' ends of sporangia. Journal of Bacteriology 121, 518523.

Hitchins, A. D. \& Slepecky, R. A. (1969). Bacterial sporulation as modified prokaryotic cell division. Nature, London 223, 804-807.

Sterlini, J. M. \& MandelstaM, J. (1969). Commitment to sporulation in Bacillus subtilis and its relationship to development of actinomycin resistance. Biochemical Journal 113, 29-37. 